\title{
SIMULTANEOUS DETERMINATION OF PIPERACILLIN AND TAZOBACTAM IN THE PHARMACEUTICAL FORMULATION TAZONAM® BY DERIVATIVE SPECTROPHOTOMETRY
}

\author{
M. INÉS TORAL*, FRANCISCA NOVA-RAMÍREZ, FALLON NACARATTE \\ Department of Chemistry, Faculty of Sciences, University of Chile, P.O. Box 653, Santiago, Chile.
}

(Received: January 11, 2012 - Accepted: February 27, 2012)

\begin{abstract}
In this paper, a simple and rapid method was developed for the simultaneous determination of Piperacillin (PIP) and Tazobactam (TAZ) by second-order derivative spectrophotometry. Water was selected as a solvent for extraction and determination of both analytes. Further studies of photo-stability and forced degradation for both drugs were also performed.

The spectral variables were optimized, a smoothing factor of $8 \cdot 10^{3}$ and a scaling factor of $1 \bullet 10^{4}$ were selected and the analytical signals were evaluated at 296.3 $\mathrm{nm}$ and $233.3 \mathrm{~nm}$ for PIP and TAZ, respectively.

The detection and quantification limits for PIP and TAZ were: $3.2 \cdot 10^{-7}$ to $9.5 \cdot 10^{-7}$ and $2.7 \cdot 10^{-7}$ to $8.9 \cdot 10^{-7} \mathrm{~mol} / \mathrm{L}$, respectively. The levels of repeatability (RSD) were $1.5 \%$ and $2.4 \%$ for PIP and TAZ respectively.

The method was applied to the pharmaceutical formulation TAZONAM NF® Wyeth. USA, with a nominal content of $4.0 \mathrm{~g}$ of PIP, $0.5 \mathrm{~g}$ of TAZ, $0.139 \mathrm{~g}$ of citric acid and $1 \mathrm{mg}$ of EDTA. A study of excipients was carried out and it was found that they do not interfere with quantification. By applying this method, a content of $3.96 \pm 0.04 \mathrm{~g}$ and $0.57 \pm 0.01 \mathrm{~g}$ was found of PIP and TAZ, respectively.
\end{abstract}

Keywords: UV-vis derivative spectrophotometry; Piperacillin; Tazobactam; Pharmaceutical formulation.

\section{INTRODUCTION}

The discovery of penicillin is one of the most important events of modern therapeutics because it has been providential for the life of man [1]. To overcome the problem of the limited activity spectrum of natural penicillins, semi-synthetic penicillins with a broader spectrum were developed [2-5]. These penicillins are effective against many gram-positive and gram-negative bacteria, but they are not resistant to penicillin.

Piperacillin is among the latest aggregates to this type of penicillin (Figure 1a) [6]. Because penicillinase is capable of hydrolyzing piperacillin and, therefore, can turn it off, a problematic situation is created. Historically, there were many infections that were untreatable and, unfortunately, many of these were quite common infections [7-9]. Because of this, the possibility was investigated of achieving a drug that contained not only piperacillin but also an active principle capable of preventing hydrolysis [10]. To do this, it was necessary to combine the semi-synthetic penicillin with a beta-lactamase inhibitor called Tazobactam, (Figure 1b) [11, 12], which together formed a commercial form called TAZONAM [NF Wyeth. USA]. This antibiotic is found in a 1:8 PIP-TAZ ratio and has been widely utilized in clinical practice in Chile and other countries like China and Greece [13] for the treatment of acute and chronic infections at the respiratory [14], renal and skin levels. Its use has even been studied at the brain level and in patients with cancer [15]. (a)

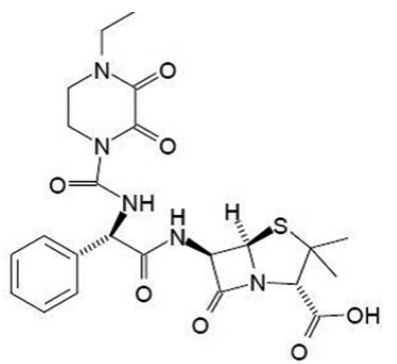

(b)

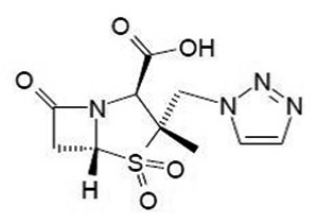

Figure 1: Structure of (a) PIP (b) TAZ.

At the present time, the PIP-TAZ determination has been made for various types of samples, such as pharmaceutical formulations, blood plasma and urine [16-18]. The methods reported for these drugs have used the HPLC technique $[17,18]$ Given the complexity of some matrices, alternatives have been proposed, such as treating the sample first with chloroform and then with acetonitrile and then the measures were carried out using the internal standard [18]. Solid phase extraction (SPE) has also been reported as an alternative with better results [19].
This paper proposes a method for the simultaneous determination of PIP-TAZ that is simple to implement in any controlled laboratory and with a spectrophotometry derivative technique, which has not been used for the PIP or TAZ analytical determination. It should be noted that results meet the analytical quality criteria and move towards the development of green chemistry [20]. This technique has been used extensively for the simultaneous determination of drug pairs which provides optimal results [21, 22, 23].

\section{EXPERIMENTAL}

\section{Instruments and apparatus}

A UV-1603PC Shimadzu spectrophotometer and quartz cells of $10 \mathrm{~mm}$ path length were used to measure the different absorbance and derivative spectra. The procedure for each measure was to correct the baseline and then record the spectra of all solutions in the range of 190-350 nm, using the same solvent as a blank, while at the same time processing the data using the software Shimadzu, kit version 3.7 (P / N 206-60570-04).

A Sartorius R 200D balance with an uncertainty of $\pm 0.01 \mathrm{mg}$ was used to weigh the samples. All procedures were conducted using a Barnstead/ Thermolyn Millipore deionizer, a vortex mixer model KMC-1300V and an

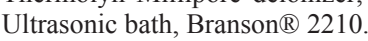

\section{Reagents}

All reagents were of analytical grade, Sodium Piperacillin and Sodium Tazobactam (Sigma - Aldrich p.a). The stock solution for PIP was of $1.0 \cdot 10^{-3}$ $\mathrm{mol} / \mathrm{L}$ for which $0.027 \pm 0.001 \mathrm{~g}$ was dissolved in $50 \mathrm{~mL}$. For TAZ, a solution of $1.0 \cdot 10^{-4} \mathrm{~mol} / \mathrm{L}$ was prepared for which $0.016 \pm 0.001 \mathrm{~g}$ of TAZ was weighed and diluted to $50 \mathrm{~mL}$ with water. A stock solution of citric acid $0.01 \mathrm{~mol} / \mathrm{L}$ was also prepared by weighing $2.104 \pm 0.1 \mathrm{~g}$ and diluting in a $1 \mathrm{~L}$ flask with distilled water.

All solutions described above were placed in an ultrasonic bath for 30 min and stored in polyethylene containers, which were previously treated with sodium hydroxide, nitric acid and distilled water.

The method was applied using the pharmaceutical formulation called TAZONAM ${ }^{\circledR}$, Wyeth Laboratory, USA containing $4.0 \mathrm{~g}$ of PIP, $0.5 \mathrm{~g}$ of TAZ, $0.139 \mathrm{~g}$ of Citric acid and $1.0 \mathrm{mg}$ of EDTA.

\section{Calibration curve}

The calibration curve was prepared from a stock solution of each drug. The concentrations that represent the calibration curve points for the PIP and TAZ were in a range of $2 \cdot 10^{-5}$ to $10 \cdot 10^{-5} \mathrm{~mol} / \mathrm{L}$ and $1 \cdot 10^{-5}$ to $5 \cdot 10^{-5} \mathrm{~mol} / \mathrm{L}$, respectively. Each solution was carried to $10 \mathrm{~mL}$ flasks with water, and then stirred with a Vortex for $1 \mathrm{~min}$. In all cases, the values for each concentration were related to the second order derivative spectra at $296.3 \mathrm{~nm}$ and $233.2 \mathrm{~nm}$ for PIP and TAZ, respectively. 
Photo-stability studies of PIP and TAZ.

Individual solutions of PIP and TAZ contained $8.0 \cdot 10^{-5}$ and $1 \cdot 10^{-5} \mathrm{~mol} / \mathrm{L}$ of each drug, respectively, were used in photo-stability studies. The solutions were exposed to direct light (sunlight), indirect light (laboratory light) and darkness. The zero-order spectra of each drug were evaluated every $30 \mathrm{~min}$ on $6 \mathrm{~h}$.

\section{Forced degradation studies of PIP and TAZ}

From the stock solution aliquots of 2 and $2.5 \mathrm{~mL}$ were taken of PIP and TAZ, respectively. Each solution was then carried to $25 \mathrm{~mL}$ flasks.

In different flasks each drug was diluted with $\mathrm{HCl} 0.01 \mathrm{~mol} / \mathrm{L}$ for acid degradation and with $\mathrm{NaOH} 0.01 \mathrm{~mol} / \mathrm{L}$ for basic degradation. In other flasks, each drug was diluted with hydrogen peroxide $0.1 \%$ for oxidative degradation and for reductive degradation $0.164 \pm 0.001 \mathrm{~g}$ of $\mathrm{Zn}$ was added and this was diluted with water. Then, for each solution, the derivative spectra were obtained after $1 \mathrm{~h}$ of constant stirring.

\section{Calibration curves of mixtures of PIP and TAZ}

In a $5 \mathrm{~mL}$ flask, aliquots of $0.5,1.0,1.5,2.0$ and $2.5 \mathrm{~mL}$ of TAZ stock solution $1 \cdot 10^{-4} \mathrm{~mol} / \mathrm{L}$ were added, and an aliquot $0.4 \mathrm{~mL}$ of PIP stock solution $1 \cdot 10^{-3} \mathrm{~mol} / \mathrm{L}$ was added to each flask. They were then diluted with water and stirred for $1 \mathrm{~min}$, obtaining for TAZ a concentration range of $1 \cdot 10^{-5}$ to $5 \cdot 10^{-5}$ $\mathrm{mol} / \mathrm{L}$ and for PIP a constant concentration of $3 \cdot 10^{-5} \mathrm{~mol} / \mathrm{L}$.

The same procedure was performed for the varying concentrations of PIP in the range of $1 \cdot 10^{-5}$ to $5 \cdot 10^{-5} \mathrm{~mol} / \mathrm{L}$, aliquots of $0.2,0.4,0.6,0.8$ and $1.0 \mathrm{~mL}$ respectively were carried to a $5 \mathrm{~mL}$ flask containing $0.5 \mathrm{~mL}$ of TAZ stock solution to reach a concentration of $1 \cdot 10^{-5} \mathrm{~mol} / \mathrm{L}$.

\section{Simultaneous determination of PIP and TAZ in synthetic samples}

Stock solutions of each drug were properly diluted to obtain solutions containing molar ratios of 1:2 to 1:10 (PIP:TAZ), including the pharmaceutical ratio $1: 8$.

In all cases, three replicates were carried out. Then the second derivative spectra were evaluated and the concentrations of each compound were calculated, allowing the determination of the corresponding values of recovery and relative standard deviation (RSD).

\section{Procedure for study of excipients}

EDTA and citric acid are reported as excipients. The pharmaceutical formulation has a content of only $1 \mathrm{mg}$ of EDTA. For this reason it was not considered as interference. Citric acid is found in greater proportion, $139 \mathrm{mg}$. For this reason, in order to study their effect, calibration curves were prepared in the presence of citric acid under two different concentrations $1 \cdot 10^{-4}$ and $7 \cdot 10^{6} \mathrm{~mol} / \mathrm{L}$. Each sample was stirred by vortex for $1 \mathrm{~min}$.

Simultaneous determination of PIP and TAZ in pharmaceutical formulation

Five ampules of TAZONAM ${ }^{\circledR}$ (Laboratory, Wyeth.USA) were weighed and powdered. A quantity of $2.5 \pm 0.01 \mathrm{mg}$ of powder was diluted in $50 \mathrm{~mL}$ of water. The solution was stirred in a sonic bath for $20 \mathrm{~min}$. This procedure was repeated for three different days.

\section{RESULT AND DISCUSSION}

\section{Solvent selection}

A preliminary qualitative study of the solubility of both drugs was conducted. It is necessary to find a solvent in which both drugs have a good solubility.Taking into account the information given in the literature and considering their structures, it was possible to select the following solvents for this study: ethanol, water and acetonitrile $[9,18]$. It was found that acetonitrile is not suitable as a solvent, since the PIP is completely insoluble. Ethanol could be used as solvent since it dissolves both. Nevertheless, water is selected because it has a higher solubility than ethanol, absorbs at $167 \mathrm{~nm}$ and does not interfere in the measurements of aqueous solutions; the analytical signals are higher and defined as for both drugs. Furthermore, the use of this solvent contributes to the development of a green chemistry.

\section{Study of photo-stability}

A study of the photo-stability of both drugs under three conditions was performed: direct light (sunlight), artificial light (laboratory light) and darkness. This study permits analysis of the effect of different types of light on the spectral behavior of both active principles, PIP and TAZ, so that precautions can be taken in handling both standards, as well as in the development of the analytical method. The above, due to exposure to light, can cause degradation of the product, changing its structures and thus alter the spectra of absorption.

The first condition to be discussed is the direct light. The solutions of both drugs were placed in direct sunlight; the ambient temperature was approximately $38^{\circ} \mathrm{C}$. Alteration in the absorption bands of both drugs was not observed. Therefore, PIP and TAZ are stable in this condition, which is consistent with information provided by the pharmaceutical laboratory Wyeth. Also, a decrease in concentrations was not observed, thus discarding the possibility that, in the presence of light, a degradation of the products occurs or that the solvent evaporates. Neither bathochromic or hypochromic effects are observed $[24,25]$ since the absorption maxima band remain constant.

In the two remaining conditions, indirect light and darkness, no changes of any kind were observed, either spectral or concentration. This is quite reasonable, considering that in sunlight, the most extreme condition, there was no alteration. Therefore, in all cases, no evidence of photochemical degradation was observed, because the spectral signals and the concentration of each agent are kept constant.

\section{Forced degradation studies of PIP and TAZ}

In the presence of $\mathrm{HCl}$, PIP presented a hyperchromic effect occurring in two peaks of absorbance and a bathochromic displacement. In the literature, for this drug three $\mathrm{pKa}$ values are reported $(2.2,4.14,16.6)$ [8], deducing that it is susceptible to changes in $\mathrm{pH}$, owing to fact that the specie is protonated. For TAZ, a hyperchromic and bathochromic effect was also produced.

In the basic hydrolysis, changes were observed for both analytes. In both cases, a significant bathochromic effect was found. This effect can be explained by the stabilization of the excited state due to the great polarity of the solvent. Since alkalizing the medium results produce an increase in $\mathrm{pH}$ which favors the donor character of the substituent.

There was no degradation observed in the presence of the reducing agent $\mathrm{Zn}$, since there is no change in the spectral bands. In the oxidative study as PIP to TAZ, the hydrogen peroxide band masks the signals. Only noise was observed because of the instability of this band in the solvent.

\section{Spectral features}

PIP and TAZ were evaluated directly against water. As can be seen in Figure 2, PIP showed an absorption peak centered at $200 \mathrm{~nm}$, but their determination is possible of 250 to $275 \mathrm{~nm}$. On the other hand; under the same conditions TAZ presents maximum absorption in the range from 200 to $220 \mathrm{~nm}$. The spectral band of PIP completely overlaps with the absorption band of TAZ, resulting in the absence of points to determine this drug. Given this, derivative spectrophotometry was used, as it allows the simultaneous determination of both drugs.

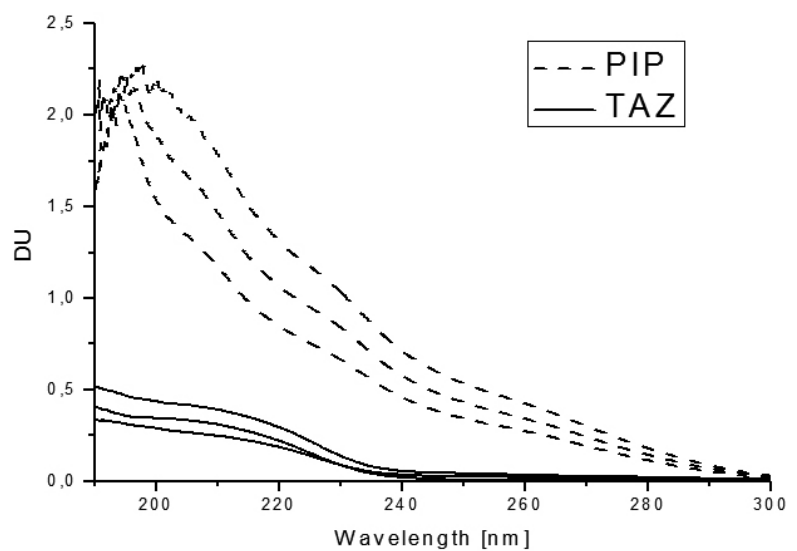

Figure 2: Classical spectrum of PIP and TAZIn water. Concentration of PIP $2 \cdot 10^{-5} ; 4 \cdot 10^{-5} ; 6 \cdot 10^{-5} \mathrm{~mol} / \mathrm{L}$ and TAZ $1 \cdot 10^{-5} ; 2 \cdot 10^{-5} ; 2 \cdot 10^{-5} \mathrm{~mol} / \mathrm{L}$.

\section{Selection of spectral variables}

The digital derivatives are processed by software coupled to the spectrophotometer [26]. Derivative spectra are obtained from computional software; the derivation is based on the mathematical model of SavitzkyGolay. This method consists in evaluating the ratio $\mathrm{A} / \Delta \lambda$ corresponding to an Absorbance column and a scanning interval $\Delta \lambda$ constant (expressed in $\mathrm{nm}$ ) using the solving least squares for differentiation, which favors the reduction of 
background noise [19]. By using this method the following spectral variables must be optimized:

- Derivative order

To select the derivative order, the maximum resolution and sensitivity must be considered without sacrificing the signal/noise ratio. Figure 3 a shows the first derivatives, all signals are present below the $\mathrm{x}$ axis and the same difficulty is presented as with classical spectra and it is not possible to use for this simultaneous determination. In the second derivative, there are areas that can be used for the simultaneous determination by zero-crossing for TAZ at $233.2 \mathrm{~nm}$ and by the graphical method for PIP at $296.3 \mathrm{~nm}$.

By increasing the derivative order there is an increased noise and a decreased sensitivity. For this reason, these derivative orders were discarded.
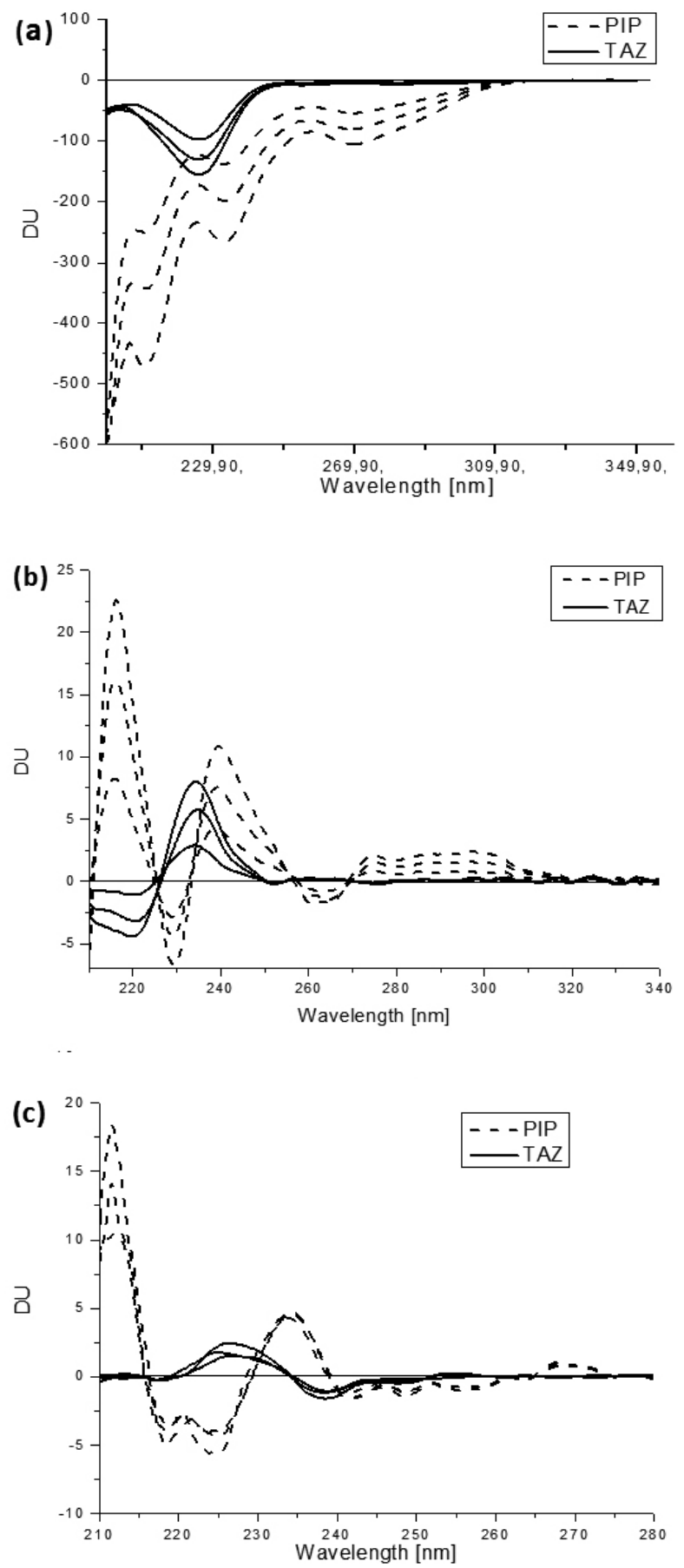

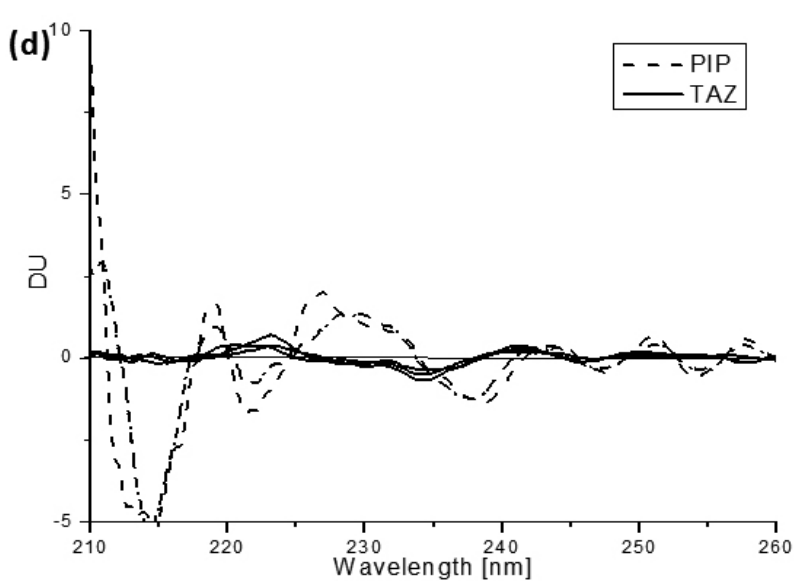

Figure 3: Derivate spectra of PIP and TAZ in water. (a) First derivative, (b) Second derivative, (c) Third derivative, (d) fourth derivative. Concentration of PIP $2 \cdot 10^{-5} ; 4 \cdot 10^{-5} ; 6 \bullet 10^{-5} \mathrm{~mol} / \mathrm{L}$ and TAZ $1 \cdot 10^{-5} ; 2 \cdot 10^{-5} ; 2 \cdot 10^{-5} \mathrm{~mol} / \mathrm{L}$ respectively.

- $\quad$ Selection of the smoothing factor

The smoothing factor is the number of points used for differentiation, which depends directly on the wavelength interval used. The measurement was limited in range from 190 to $350 \mathrm{~nm}$, since outside it there is no absorption. The software identifies the factors that can be used [27], these are: $1 \bullet 10^{3}, 2 \cdot 10^{3}$, $4 \cdot 10^{3}$ and $8 \cdot 10^{3}$ (Figure 4 ). As the factor increases, the heights of the derivatives decrease and also a clear reduction of noise is observed. Factor $4 \cdot 10^{3}$ shows a good signal, but the signals are not proportional to the concentration so it does not have analytical usefulness. In order to have a high signal/noise, good sensitivity and definition, a smoothing factor of $8 \bullet 10^{3}$ was selected.
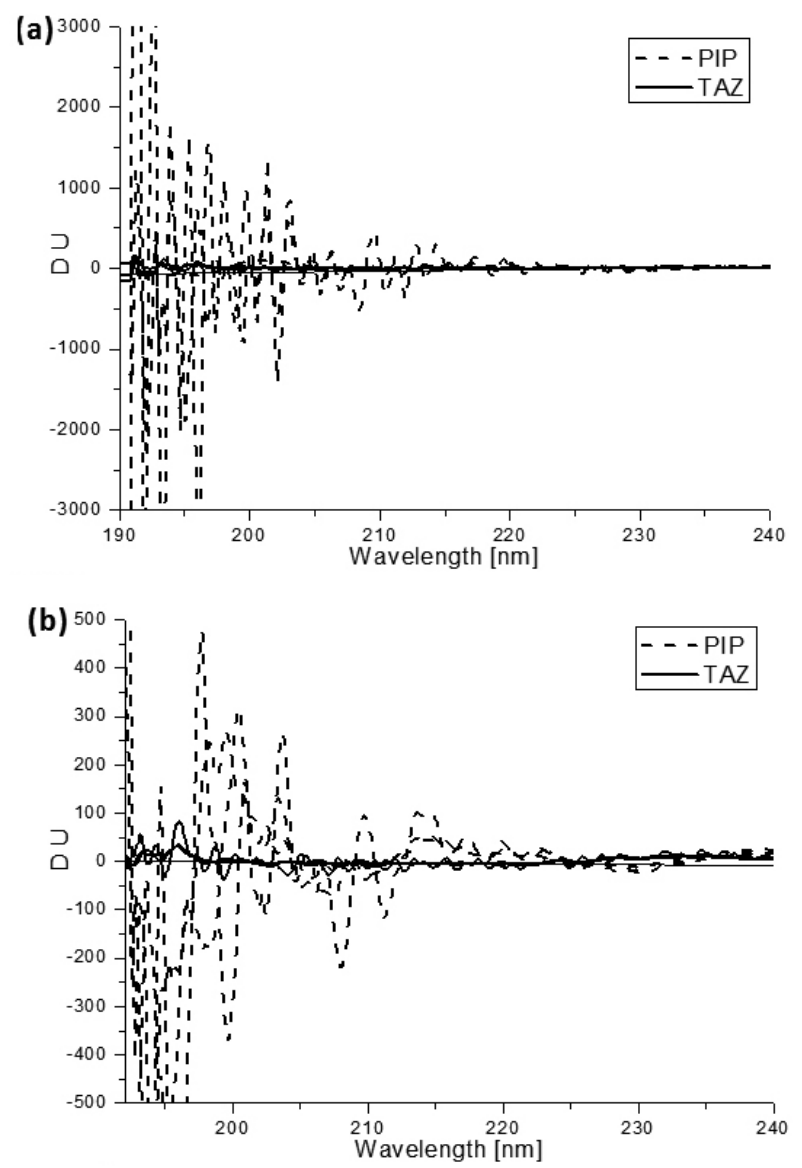

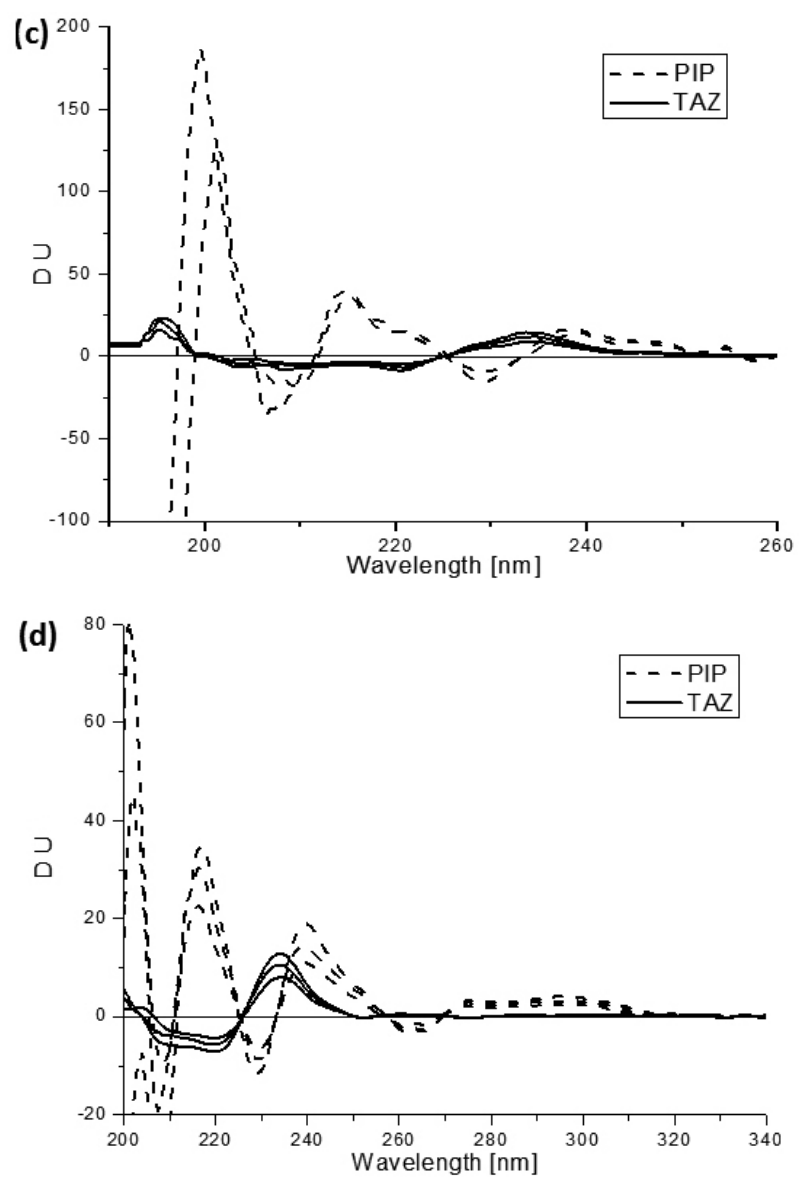

Figure 4: Effect of the smoothing factor on second derivative of PIP and TAZ (a) $1 \bullet 10^{3}$, (b) $2 \cdot 10^{3}$, (c) $4 \bullet 10^{3}$, (d) $8 \bullet 10^{3}$. Concentration of PIP $2 \bullet 10^{-5} ; 4 \bullet 10$ $5 ; 6 \cdot 10^{-5} \mathrm{~mol} / \mathrm{L}$ and TAZ $1 \cdot 10^{-5} ; 2 \cdot 10^{-5} ; 2 \cdot 10^{-5} \mathrm{~mol} / \mathrm{L}$ respectively

\section{- $\quad$ Selection of the scale factor}

Amplifies the signal between 1 to $10^{11}$ times, increases the analytical signal and the background noise in the same proportion [28]. This factor only affects the attainment of a good reading of the analytical signal since it does not increase sensitivity.

This factor is used to amplify the analytical signal and the background noise in the same proportion [29]. It is necessary that a balance exists between signal amplification and the distorting effect on the spectral bands, because if the signal is amplified too much, it can distort, causing a misreading. It is for this reason that for the simultaneous determination a scaling factor of $1 \cdot 10^{4}$ was selected that gives a reading with three decimals without producing distorting effects.

\section{- Analytical wavelength selection}

As can be seen in Figure 3b, the second order derivatives present characteristic zones for quantify PIP, because in this derivative spectral at $225.5 \mathrm{~nm}$ and in a range of $275-350 \mathrm{~nm}$ TAZ does not absorb despite being in high concentrations and the DU can only be attributed to PIP. This latter range was selected to quantify PIP

In addition, a good linearity of the signals was observed. The method used for determination was the graphic method. Within the range, $296.3 \mathrm{~nm}$ was selected because it has more height and definition, which favors sensitivity and a lower background noise.

Similar analysis is performed to determine TAZ, since there are two possible zero-crossing at $210.5 \mathrm{~nm}$ and $233.2 \mathrm{~nm}$. The first one was not selected as an analytical wavelength because it is a not suitable point, since it is very near to the lower limit of the technique and has a greater error, since in this area there is a significant noise spectrum, which decreases accuracy. In contrast, the $233.2 \mathrm{~nm}$ does not vary and the signals have a good linearity. According to this analysis, the simultaneous determination of PIP and TAZ can be carried out at $296.3 \mathrm{~nm}$ by the graphical method and $233.2 \mathrm{~nm}$ by zero-crossing, respectively.

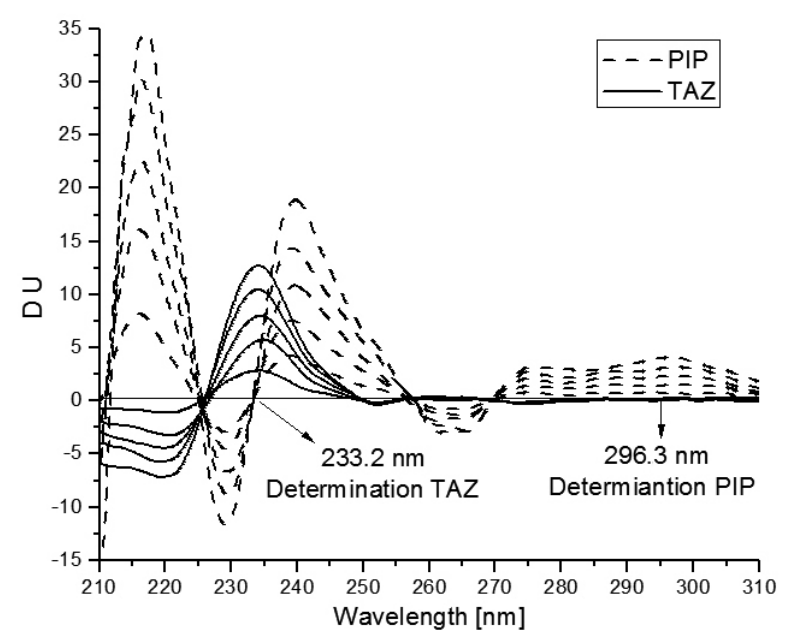

Figure 5. Second derivative of PIP and TAZ in water. Concentration of PIP $2 \cdot 10^{-5} ; 4 \cdot 10^{-5} ; 6 \cdot 10^{-5} \mathrm{~mol} / \mathrm{L}$ and TAZ $1 \cdot 10^{-5} ; 2 \cdot 10^{-5} ; 2 \cdot 10^{-5} \mathrm{~mol} / \mathrm{L}$ respectively.

\section{Analytical features}

Calibration curves were obtained by plotting the second derivative values for PIP, DU $(\lambda=296.3 \mathrm{~nm})$ and TAZ, DU $(\lambda=233.3 \mathrm{~nm})$, using a smoothing factor of 8,000 , a scale factor of 100,000 and a $\Delta \lambda$ of $150 \mathrm{~nm}(200$ to $350 \mathrm{~nm})$, versus the respective drug concentrations. Linear regression equations were obtained from calibration curves.

$$
\begin{array}{lcr}
\text { PIP } & \mathrm{Y}=4.3 \cdot 10^{4} \mathrm{X}+0.0027 & \mathrm{R}=0.9996 \\
\text { TAZ } & \mathrm{Y}=2.7 \cdot 10^{5} \mathrm{X}-0.0104 & \mathrm{R}=0.9998
\end{array}
$$

The analytical features were obtained using the criteria given by ICH Harmonized Tripartite Guideline, to calculate the detection and quantification limits (DL, QL). The following equations were used: $3.3 \mathrm{\sigma} / \mathrm{S}$ and $10 \mathrm{\sigma} / \mathrm{S}$, respectively, where $S$ is the slope of the calibration curve and $\sigma$ the standard deviation corresponding to the response of 11 blanks. The repeatability, expressed as relative standard deviation (RSD), was obtained by using 9 standard samples containing a mixture of the drugs in proportion 1:2, 1:6 and 1:8 (PIP:TAZ). The determination range was defined between the $\mathrm{QL}$ and the loss of linearity. In this context, it is possible to obtain an acceptable linearity degree with accuracy and precision in the results when the proposed method is applied. The results are show in the Table 1.

\begin{tabular}{|c|c|c|}
\hline Parameter & PIP & TAZ \\
\hline $\begin{array}{l}\text { Quantification limit } \\
(\mathrm{mol} / \mathrm{L})\end{array}$ & $9.5 \cdot 10^{-7}$ & $8.9 \cdot 10^{-7}$ \\
\hline Detection limit $(\mathrm{mol} / \mathrm{L})$ & $3.2 \cdot 10^{-7}$ & $2.7 \cdot 10^{-7}$ \\
\hline $\begin{array}{l}\text { Determination range } \\
(\mathrm{mol} / \mathrm{L})\end{array}$ & $9.5 \cdot 10^{-7}-5.0 \cdot 10^{-3}$ & $8.9 \cdot 10^{-7}-1.0 \cdot 10^{-4}$ \\
\hline Repeatability (RSD\%) & $<2.0$ & $<2.0$ \\
\hline Linear regression & $\mathrm{Y}=4.3 \cdot 10^{4} \mathrm{X}+0.0027$ & $\begin{array}{c}\mathrm{Y}=2.7 \cdot 10^{5} \mathrm{X}- \\
0.0104\end{array}$ \\
\hline Correlation coefficient & 0.9998 & 0.9996 \\
\hline
\end{tabular}

Table 1: Analytical parameters.

\section{Recovery in synthetic samples}

A study was conducted to observe the effect of concentration on the simultaneous quantification of both drugs, according to procedure. Because the pharmaceutical formulation contains a relation of PIP and TAZ, it was necessary to establish the proportions in which an analyte can be measured precisely and accurately in the presence of another. Twelve replicas for each synthetic samples were prepared from stock solutions, in order to evaluate the accuracy of the method, recovery percentages were determined for replicas in which the pharmaceutical proportion of 1:8 ratio was included. For PIP and TAZ, $98-100 \%$ and $97-103 \%$ were obtained, respectively, which represent a good accuracy of the method. In order to evaluate the accuracy, the relative standard deviation (RSD) was calculated. For PIP and TAZ, this was $1.5 \%$ and 
$2.0 \%$, respectively. In both cases, a RSD $<2.0 \%$ was found. Both results show good repeatability and reproducibility of the proposed method.

\section{Effects excipients studies}

The pharmaceutical formulation reports as excipients $139 \mathrm{mg}$ of citric acid (CA) and $1 \mathrm{mg}$ of EDTA. Among the excipients, CA was only considered because it is found in greater proportion compared with EDTA. CA also has chromophores groups which produce an absorption band in the UV-vis., where its maximum is located around $240 \mathrm{~nm}$ [30]. For this reason interfere with the determination of TAZ and even of PIP.

To do this, calibration curves were prepared for PIP and TAZ in the presence of CA in concentrations, $1 \cdot 10^{-4}$ and $7 \cdot 10^{-6} \mathrm{~mol} / \mathrm{L}$, respectively. These spectral bands were compared in presence and absence of CA $1 \cdot 10^{-4}$. The results suggest that this amount of acid would affect the spectral bands. In the case of TAZ, it does not produce this effect because its pKa is very low [26].

On the other hand, a second calibration curve was prepared with acid to $7 \cdot 10^{-6} \mathrm{~mol} / \mathrm{L}$. Just as above, the calibration curve was compared in the absence of acid and there was no difference in the absorbance measurements, analytical wavelengths for PIP and TAZ not differ. Since the concentration of CA corresponds to reported in the pharmaceutical formulation TAZONAM®, it possible to stablish that $\mathrm{CA}$ does not behave as interfering in this simultaneous determination

\section{Application of the proposed method}

The method was applied in real samples of the drug TAZONAM $\AA$. A mass of $3.96 \pm 0.04 \mathrm{~g}$ was found for PIP and $0.57 \pm 0.01 \mathrm{~g}$ for TAZ. Both results were compared with the reported nominal content, PIP $4 \mathrm{~g}$ and TAZ $0.5 \mathrm{~g}$. For PIP there is no significant difference between the found value and the reported value in the drug ( $\mathrm{t}$ test gave a $99 \%$ confidence).

Instead, TAZ has a greater difference, possibly because the content reported by the drug may be less than the real amount of TAZ in TAZONAM $₫$. Note that the allowable range for PIP and TAZ is between 90 and $110 \%$ of the amount reported; that is because TAZ, despite having a deviation from the nominal content, is under the limit allowed by the FDA [8].

\section{CONCLUSIONS}

An accurate, precise, direct and inexpensive second derivative spectrophotometry method has been developed for the simultaneous determination of PIP and TAZ in pharmaceutical formulations.

The success of the recovery of nominal content for each drug from the pharmaceutical formulation shows that there was no matrix effect.

The stability study of the photo-stability shows that, under ambient light and dark, no artificial alterations occur in the structure of PIP and TAZ because the bands do not exhibit changes in intensity and form, thus ruling out photochemical degradation. Forced stability with reducing agents found no change in the absorption bands. However, there are major changes in the absorption bands under basic and acid hydrolysis.

The contribution of this work is the development of a method for the simultaneous determination proposal, which has not been reported and can be considered as a good analytic proposal.

\section{ACKNOWLEDGEMENTS}

The authors thank the project FONDECYT N 1100103 for financial support for this investigation.

\section{REFERENCES}

1. Hamilton-Miller, J.M.T. Int J Antimicrob. Agents. 31, 189-192, (2008).

2. L. Li, Z. Jiang, L. Xia , Jian-Zhong Shen, Lei Dai, Yang Wang, Si-Yang Huang, Cong-Ming Wu. Vet Microbiol. 144, 505-510, (2007)

3. Z. Zhiyong, L. Xiaoju, L. Yanbin, Y. Yao, Y. Rujia, F. Xueqin, H Wenxiang, C. Sufang, Y. Zebo, Z. Xingping, D. Minggang, X. Peiyuan, Z. Weiming, J. Xianghui, Z. Hongwen, C. Yongchuan, Y. Fei, N. Zongzan. Int J Antimicrob Agents. 26, 22-27, (2005).

4. C.W. Stratton MD. Antimicrob infect dis newsl.15, 67-72, (2001).

5. I. Ito, S. Kadowaki, N.Tanabe, A. Haruna, M. Kase, Y. Yasutomo, M. Tsukino, A. Nakai, H. Matsumoto, A. Niimi, K. Chin, S. Ichiyama, M. Mishima . Pulm Pharmacol Ther. 23, 403-410, (2010).

6. G. Moet, A. Watters, S. Sader, R. Jones. Diagn Microbiol Infect Dis. 65, 319-322, (2009).

7. W. Lau, D. Mercer, K. Itani, D. Nicolau, J. Kuti, D.Mansfield, A. Dana. Antimicrob Agents Chemother. 50, 3556-3566, (2006).

8. R. Nau, M Kinzing-Schippers, F.Sorgel, S. Schinschke, R. Rossing, C. Muller, H. Kolenda, H. W. Prange Antimicrob. Agents Chemother. 41, 987-991, (1997).

9. D. S. Burgess, T.Waldrep. Clin Ther. 24, 1090-1104, (2002).

10. Q. Liu, K. Rand, H.Derendorf. Int J Antimicrob Agents. 23, 494-497, (2004).

11. S.Kotapati, J. L. Kuti, E. C. Geissler, C.H .Nightingale, D. P Nicolau. Int Crit Care Nurs. 21, 87-93, (2005).

12. D. S. Burgess, T.Waldrep. Clin Ther.24, 1090-1104, (2002).

13. C. Xia, Y.Xiong. Biomed.Chrometogr. 21, 680-686, (2007).

14. N. London, C.J. Thomson, S.G.B. Amyes, E. Stobberingh. FEMS Immunol Med Microbiol. 11, 107-111, (1995).

15. N. S. Viana Jr., L. M. Moreira-Campos, C. D. Vianna-Soares. IlFarmaco, 60, 900-905, (2005).

16. V. Augey, P. Grosse, G. Albert, M. Audran, F. Bressolle J Chromatogr B Biomed Sci Appl. 682, 125-136, (1996).

17. S. C. Marcelos, H.A.Archontaki. J.pharmaceutical and bio. Analysis. 29, 787-793, (2002)

18. R Trittler, M Ehrlich, T.J Galla, R.E. J. Chromatogr B. 775,127-132, (2002).

19. J.Karpinska, Anal Chim Acta. 518, 1-24,(2004).

20. P. T. Anastas, R. L. Lankey. Green Chem. 2, 289-295, (2000).

21. M. Inés Toral, N. Lara, A. Tapia, C. Torres, P. Richter. J. Chil. Chem. Soc. 47, 241-251, (2002)

22. M. Inés Toral, J. Rivas, M. Saldías, C. Soto, S. Orellana. J. Chil. Chem. Soc. 53,1543-1547, (2008)

23. C. Soto, D. Contreras, M. Inés Toral, L. Basaez, J. Freer. J. Chil. Chem. Soc. 54, 113-118, (2009)

24. Yang, Cheng-Hsien, Chen, Chun-Hung, Sun, I-Wen. Polyhedron. 25 , 2407-2414, (2006).

25. A. Lababpour, C. Lee. J. Biosci Bioeng.101, 104-110, (2006)

26. G. J. Moet, A. Watters, H. S. Sader, R. N. Jones. Diagn. Microbiol. Infect. Dis. 65, 319-322, (2009).

27. M. Inés Toral, S. Orellana, M. Saldías.Quim.Nova.32, 257-262, (2009)

28. C. Bosch, F. Sánchez. Anal Chim Acta.518, 1-24, (2004).

29. A. Lababpour, C.Lee. J. Biosci Bioeng.101, 104-110, (2006).

30. M. Szyper, P Zurman. Anal Chim Acta. 85, 357-373, (1976). 